Tohoku J. exp. Med., 1976, 119, 171-183

\title{
Errors of Parameters of Distribution Functions for Spherical Bodies Stochastically Estimated on a Random Test Plane
}

\author{
Norio SuWA \\ Department of Pathology, Tohoku University School of Medicine, \\ Sendai
}

Suwa, N. Errors of Parameters of Distribution Functions for Spherical Bodies Stochastically Estimated on a Random Test Plane. Tohoku J. exp. Med., 1976, 119 (2), 171-183 — The parameters of some distribution functions for the radius of spherical bodies randomly dispersed in a three-dimensional space can be estimated on a random test plane of unit surface area. In this estimation the set of measured $N a O, \bar{\delta}$ and $\left(\overline{\delta^{2}}\right)$ or that of $N \lambda o, \bar{\lambda}$ and $\left(\overline{\lambda^{2}}\right)$ is used. They are number of circles, arithmetical mean and secondary moment of circle diameters or number of chords delivered by intersection of a test line of unit length with eircles, arithmetical mean and secondary moment of chord length, respectively. Provided that the region of measurement is sufficiently large, intraregional errors of these quantities expressed as the squares of coefficient of variation $C$ are approximately: $[C(N a o)]_{w}{ }^{2}=1 / \bar{N} a o,[C(\delta)]_{w}{ }^{2}=\left(32 / 3 \pi^{2}\right)\left(Q_{3} / Q_{2}{ }^{2}\right)(1 / \bar{N} a o)$ and $\left[C\left(\overline{\delta^{2}}\right)\right]_{w}{ }^{2}$ $=(6 / 5)\left(Q_{5} / Q_{3}{ }^{2}\right)(1 / \bar{N} a o) ;[C(N \lambda o)]_{w}{ }^{2}=1 / M \bar{N} \lambda o,[C(\bar{\lambda})]_{\omega}{ }^{2}=(9 / 8)\left(Q_{4} Q_{2} / Q_{3}{ }^{2}\right)(1 / M \bar{N} \lambda o)$ and $\left[C\left(\overline{\lambda^{2}}\right)\right]_{w}{ }^{2}=(4 / 3)\left(Q_{6} Q_{2} / Q_{4}{ }^{2}\right)(1 / M \bar{N} \lambda o)$. In these expressions $Q_{n}$ is a quotient defined by $\left(\bar{D}^{n}\right) / \bar{D}^{n}, D$ and $n$ being sphere diameter and a positive integer, respecitvely. The first three expressions may be used in these forms as the errors for the total region containing spheres. In the second three expressions $M$ is the number of random test lines of unit length. When $M$ is small, they can stand for the errors of the total region. In the case of large $M$, however, interregional errors or errors of sampling have to be added to them. The geometrical parameter of a distribution function of $D$ is estimated from $\left(\overline{\delta^{2}}\right) / \bar{\delta}^{2}$ or $\left(\overline{\lambda^{2}}\right) / \bar{\lambda}^{2}$. The error of this ratio $W$ is given by: $[C(W)]^{2}=\left[C\left(\overline{X^{2}}\right)-2 C(\bar{X})\right]^{2}$, where $X$ stands for $\delta$ or $\lambda . \longrightarrow$ stochastic method; sphere distribution; distribution function; error

In a previous report, Suwa et al. (1976) described stochastic methods to estimate the parameters of some distribution functions assumed for spherical bodies of different sizes randomly dispersed in a three-dimensional space. Either the diameter of circles on a random test plane or the length of chords delivered by intersection of random test lines with the circles may be used for the estimation. Stochastic methods are always associated with errors of estimates. In a practical application of the methods, it is therefore required to calculate them. It will make possible to evaluate the reliability of estimation and contribute to a reasonable design of measurements. In the present study, attempts are made to derive practically useful mathematical expressions for the errors. The derivation presupposes the stochastic methods of the previous report, which are only referred to

Received for publication, January $23,1976$. 
without detailed comments in this connection. Important symbols used in the mathematical treatment are not only explained in the text but also summarized in an attached table. Some theorems of statistics are presented in Appendix and are indicated in the text with $A$ and a number like (A.1).

\section{Mathematroal Treatments}

General Structure of Errors. In the majority of biological objects, the number of spherical bodies contained in the total region is so large that they cannot be submitted to measurement as a whole. Consequently, a region of relatively limited size is usually taken out of the total region, and a certain quantity in reference to the total region is estimated from the measurement on the selected region. The error of estimation is then composed of two independent components. One of them arises from the sampling process. Provided that the distribution of spherical bodies is randomized, the number of spherical bodies or any other quantity in reference to them is subject to variation according to the regions selected, even if they are of the same size. The error of this origin is called "interregional error" in the present study.

The other component is due to stochastic estimation of a certain quantity of spheres within a selected region. If the region is traversed by a random sectional plane, the circles on the plane are different in each trial. The chords delivered by intersection of test lines drawn randomly on the test plane are also susceptible of variation. The error of this nature is called "intraregional error" in the following descriptions.

The error may be expressed in a number of different ways. In the present study, the square of coefficient of variation $C$ or of the ratio of standard deviation to arithmetical mean is used in view of its easy mathematical treatment. Now, let the estimated quantity be $x$, and let the quantity referred to the total region be indicated by *. Then there is the following important relation

$$
\left[C(x)^{*}\right]^{2}=[C(x)]_{w}{ }^{2}+[C(x)]_{g}{ }^{2},
$$

where the subscripts $w$ and $g$ denote intraregional and interregional errors, respectively. Expression (1) means that the error of $x$ in reference to the total region is the sum of intraregional and interregional errors of $x$. This additive principle makes it possible to compile the error for the total region from separate calculation of intraregional and interregional errors.

Preliminary Derivations. Because spherical bodies are distributed in a threedimensional space, the region of measurement must primarily be also a threedimensional one. When the diameter of circles on a test plane is utilized, a cube with edges of unit length is taken as the region of measurement. The size of the actual cube may be arbitrarily chosen, but the length of its edges has to be treated as the unit. If the chord length is used, however, it is rather practical to take a large plane as the total region. If a very large three-dimensional space is traversed by a very large sectional plane, the circles on the plane will provide an unbiased 
information about the sphere distribution in the three-dimensional space. From this large plane a plane of adequate size is taken as a region of measurement, and its surface area is treated as the unit. On this selected region test lines are drawn in a sufficiently large number in order to deliver a sufficiently large number of chords.

Now, the mathematical derivations for errors start from the following relations

$$
\begin{aligned}
& \operatorname{In}(\delta)=\frac{\sqrt{\pi}}{2}\left[n \Gamma\left(\frac{n}{2}\right) /(n+1) \Gamma\left(\frac{n+1}{2}\right)\right] Q_{n+1} \bar{D}^{n+1} N v o \\
& \operatorname{In}(\lambda)=\frac{\sqrt{\pi}}{2}\left[n \Gamma\left(\frac{n}{2}\right) /(n+1) \Gamma\left(\frac{n+1}{2}\right)\right] Q_{n+1}^{\prime} \bar{\delta}^{n+1} N a o .
\end{aligned}
$$

In these expressions $\delta$ and $\lambda$ are the diameter of a circle on the test plane and the length of a chord delivered from a test line by intersection with a circle, respectively; and $\bar{D}$ and $\bar{\delta}$ are the arithmetical means of sphere diameter $D$ and circle diameter $\delta$, respectively. $\operatorname{In}(\delta)$ and $\operatorname{In}(\lambda)$ are defined by the following integrations

$$
\begin{aligned}
& \operatorname{In}(\delta)=N a o \int_{0}^{\infty} \delta^{n} p(\delta) \mathrm{d} \delta \\
& \operatorname{In}(\lambda)=N \lambda o \int_{0}^{\infty} \lambda^{n} p(\lambda) \mathrm{d} \lambda,
\end{aligned}
$$

and

in which $n$ is 0 or a positive integer, $p(\delta)$ and $p(\lambda)$ are probability distribution functions for $\delta$ and $\lambda$, respectively. $Q_{n}$ and $Q_{n}{ }^{\prime}$ are quotients determined by

$$
\begin{aligned}
& Q_{n}=\left(\overline{D^{n}}\right) / \bar{D}^{n}=\int_{0}^{\infty} D^{n} p(D) \mathrm{d} D / \bar{D}^{n} \\
& Q_{n}{ }^{\prime}=\left(\overline{\delta^{n}}\right) / \bar{\delta}^{n}=\int_{0}^{\infty} \delta^{n} p(\delta) \mathrm{d} \delta / \bar{\delta}^{n} .
\end{aligned}
$$

Nvo, Nao and Nגo are the total numbers of spheres in a unit volume, of circles on a unit surface area, and of chords per test line of unit length, respectively.

The expression (2) was derived in the report of Suwa et al. (1976), and (3) is obtained in a similar way. Because the distribution of sphere diameters is generally different from that of circle diameters on a sectional plane, $Q_{n}$ cannot be immediately introduced into (3), and a new quotient $Q_{n \prime}^{\prime}$ is defined by the distribution of $\delta$. The relation between $Q_{n}$ and $Q_{n}{ }^{\prime}$ is determined by the following expression.

$$
\begin{aligned}
Q_{n}^{\prime} & =\frac{\left[\operatorname{In}(\delta) / I_{0}(\delta)\right]}{\left[I_{1}(\delta) / I_{0}(\delta)\right]^{n}} \\
& =2^{2 n-1} \sqrt{\pi}\left[n \Gamma\left(\frac{n}{2}\right) /(n+1) \Gamma\left(\frac{n+1}{2}\right)\right] Q_{n+1} /\left(\pi Q_{2}\right)^{n} .
\end{aligned}
$$

The next step is a transformation of (2) and (3). The expectations of circle 
number $N a 0$ and chord number $N \lambda o$ are now written as $\bar{N} a o$ and $\bar{N} \lambda o$, respectively. There are then the following relations.

$$
\bar{N} a o=\bar{D} N \text { vo, } \bar{N} \lambda o=\bar{\delta} N_{a o}
$$

and

$$
\left(\overline{\delta^{n}}\right)=\operatorname{In}(\delta) / \bar{N} a o, \quad\left(\overline{\lambda^{n}}\right)=\operatorname{In}(\lambda) / \bar{N} \lambda o,
$$

in which $\left(\overline{\delta^{n}}\right)$ and $\left(\overline{\lambda^{n}}\right)$ are the $n$-th moments of $\delta$ and $\lambda$, respectively. With (8) (9) and (10), (2) and (3) are transformed into

$$
\left(\overline{\delta^{n}}\right)=\frac{\sqrt{\pi}}{2}\left[n \Gamma\left(\frac{n}{2}\right) /(n+1) \Gamma\left(\frac{n+1}{2}\right)\right] Q_{n+1} \bar{D}^{n}
$$

and

$$
\left(\overline{\lambda^{n}}\right)=2^{2 n+1} Q_{n+2} \bar{\delta}^{n} /(n+2) \pi^{n} Q_{2}{ }^{n+1} \text {. }
$$

From (11) and (12)

$$
\begin{aligned}
& \bar{\delta}=(\pi / 4) Q_{2} \bar{D} \\
& \left(\overline{\delta^{2}}\right)=(2 / 3) Q_{3} \bar{D}^{2} \\
& \bar{\lambda}=(8 / 3 \pi)\left(Q_{3} / Q_{2}{ }^{2}\right) \bar{\delta}
\end{aligned}
$$

and

$$
\left(\overline{\lambda^{2}}\right)=\left(8 / \pi^{2}\right)\left(Q_{4} / Q_{2}{ }^{3}\right) \bar{\delta}^{2}
$$

are immediately derived. These relations are used in the subsequent derivations of errors together with (2), (3) and (8).

In the previous report of Suwa et al. (1976) the geometrical parameter of a theoretical distribution function for sphere radii is estimated from $\left(\overline{\delta^{2}}\right) / \bar{\delta}^{2}$ or $\left(\overline{\lambda^{2}}\right) / \bar{\lambda}^{2}$. The scalar parameter is then calculated with the aid of $\bar{\delta}$ or $\bar{\lambda}$. In the calculation of the total number of spheres in a unit volume $\bar{N}$ ao or $\bar{N} \lambda o$ is further utilized. Consequently, the errors in the estimation of $N a o, \bar{\delta}$ and $\left(\overline{\delta^{2}}\right)$ and those of $N \lambda o, \bar{\lambda}$ and $\left(\overline{\lambda^{2}}\right)$ are to be derived, as the preliminary steps for the calculation of the errors of parameters.

Interregional Error. Interregional error of Nao arises from the variation of the number and size of spheres contained in a region of measurement. Owing to the relation of $\bar{N} a_{0}=\bar{D} N v_{0}$ and because $\bar{D}$ and $N v o$ are independent of each other, one obtains according to (A. 20) and (A. 11)

$$
\begin{aligned}
{[C(N a o)]_{g}{ }^{2} } & =C(N v o)^{2}+C(\bar{D})^{2}=\left[1+C(D)^{2}\right] / N v o \\
& =Q_{2} / N v o=(4 / \pi)(\bar{\delta} / \bar{N} a o) .
\end{aligned}
$$

On the other hand, (13) and (14) show that interregional errors of $\vec{\delta}$ and $\left(\overline{\delta^{2}}\right)$ are influenced only by the variation of $\bar{D}$, because $Q_{n}$ may be approximately regarded as a constant, so that they are expressed as

$$
\begin{aligned}
{[C(\bar{\delta})]_{g}{ }^{2} } & =C(\bar{D})^{2}=C(D)^{2} / N v o \\
& =\left(Q_{2}-1\right) / N v o=(4 / \pi)\left[1-\left(1 / Q_{2}\right)\right](\bar{\delta} / \bar{N} a o) \\
{\left[C\left(\overline{\delta^{2}}\right)\right]_{g}{ }^{2} } & =4\left(Q_{2}-1\right) / N v o \\
& =(16 / \pi)\left[1-\left(1 / Q_{2}\right)\right](\bar{\delta} / \bar{N} a o) .
\end{aligned}
$$


In the derivation of (19), (A. 15) is referred to.

Interregional ercors of $N \lambda a, \bar{\lambda}$ and $\left(\overline{\lambda^{2}}\right)$ can also be calculated in the same way, and the results are

$$
\begin{aligned}
& {[C(N \lambda o)]_{g}{ }^{2}=Q_{2}{ }^{\prime} / N a o=(4 / \pi)(\bar{\lambda} / \bar{N} \lambda o)} \\
& \begin{aligned}
{[C(\bar{\lambda})]_{g}{ }^{2} } & =\left(Q_{2}{ }^{\prime}-1\right) / N a o \\
& =(4 / \pi)\left[1-\left(3 \pi^{2} / 32\right)\left(Q_{2}{ }^{2} / Q_{3}\right)\right](\bar{\lambda} / \bar{N} \lambda o)
\end{aligned}
\end{aligned}
$$

and

$$
\begin{aligned}
{\left[C\left(\overline{\lambda^{2}}\right)\right]_{\mathrm{g}}{ }^{2} } & =4\left(Q_{2}{ }^{\prime}-1\right) / N a o \\
& =(16 / \pi)\left[1-\left(3 \pi^{2} / 32\right)\left(Q_{2}{ }^{2} / Q_{3}\right)\right](\bar{\lambda} / \bar{N} \lambda o) .
\end{aligned}
$$

Intraregional Error. Intraregional error of $N a 0$ is the variation of the number of circles on a test plane of unit surface area in each trial. If the region of measurement is sufficiently large, so that the probability of a sphere to be sectioned by a random test plane is very small, Nao will follow the Poisson distribution. The expectation $\bar{N} a o$ of $N a o$ is then equal to the variance $\operatorname{Var}(N a o)$ of $N a o$, and one obtains

and

$$
\bar{N} a o=\operatorname{Var}(N a o)=\bar{D} N v o
$$

$$
[C(N a o)]_{w^{2}}=1 / \bar{D} N v o=1 / \bar{N} a o .
$$

For the derivation of intraregional errors of $\bar{\delta}$ and $\left(\overline{\delta^{2}}\right)$, take at first a single sphere of $D_{j}$ in diameter. The sphere will deliver circles of different sizes according to its relative position to a test plane. The diameter of the circles contributed by this sphere is denoted by $\delta_{j}$ and the expectation and variance of $\delta_{j}$ by $\bar{\delta}_{j}$ and $\operatorname{Var}\left(\delta_{j}\right)$, respectively. Applying (2) to a single sphere one obtains

$$
\begin{aligned}
& \bar{\delta}_{j}=(\pi / 4) D_{j}{ }^{2} \\
& \overline{\left(\delta_{j}^{2}\right)}=(2 / 3) D_{j}{ }^{3} \\
& \overline{\left(\delta_{j}{ }^{4}\right)}=(8 / 15) D_{j}{ }^{5} .
\end{aligned}
$$

and

On account of $(A, 3)$ the variances of $\delta_{j}$ and $\delta_{j}^{2}$ are

and

$$
\begin{aligned}
& \operatorname{Var}\left(\delta_{j}\right)=(2 / 3) D_{j}^{3}-(\pi / 4)^{2} D_{j}{ }^{4} \\
& \operatorname{Var}\left(\delta_{j}^{2}\right)=(8 / 15) D_{j}^{5}-(2 / 3)^{2} D_{j}{ }^{6} .
\end{aligned}
$$

Extending the above results to the total group of spheres of different sizes

and

$$
\begin{aligned}
& (\overline{\Sigma \delta})=\sum_{j}(\pi / 4) D_{j}^{2}=(\pi / 4) \bar{D}^{2} Q_{2} N v o \\
& \left(\overline{\Sigma \delta^{2}}\right)=\sum_{j}(2 / 3) D_{j}^{3}=(2 / 3) \bar{D}^{3} Q_{3} N v o \\
& \operatorname{Var}(\delta)=(2 / 3) \bar{D}^{3} Q_{3} N v o-(\pi / 4)^{2} \bar{D}^{4} Q_{4} N v o \\
& \operatorname{Var}\left(\delta^{2}\right)=(8 / 15) \bar{D}^{5} Q_{5} N v o-(2 / 3)^{2} \bar{D}^{6} Q_{6} N v o
\end{aligned}
$$

are obtained. The errors of $\bar{\delta}$ and $\left(\overline{\delta^{2}}\right)$ are equal to those of $(\overline{\Sigma \delta})$ and $\left(\overline{\Sigma \delta^{2}}\right)$ respectively according to $(A .11)$ and are consequently

$$
[C(\bar{\delta})]_{w}{ }^{2}=\left(32 / 3 \pi^{2}\right)\left(Q_{3} / Q_{2}{ }^{2}\right)(1 / \bar{N} a o)-\left(Q_{4} / Q_{2}{ }^{2}\right)(1 / N v o)
$$




$$
\left[C\left(\overline{\delta^{2}}\right)\right]_{w}{ }^{2}=(6 / 5)\left(Q_{5} / Q_{3}{ }^{2}\right)(1 / \bar{N} a 0)-\left(Q_{6} / Q_{3}{ }^{2}\right)(1 / N v o) .
$$

When the region of measurement is sufficiently large, Nvo is very much larger than Nao, so that the second terms on the right sides of (34) and (35) may be deleted. In this case, interregional errors are also negligibly small in comparison with intraregional errors. The final expressions of the errors of $N a o, \delta$ and $\left(\overline{\delta^{2}}\right)$ in reference to the total region are then

and

$$
\begin{aligned}
& {\left[C(N a o)^{*}\right]^{2}=1 / \bar{N} a o} \\
& {\left[C(\bar{\delta})^{*}\right]^{2}=\left(32 / 3 \pi^{2}\right)\left(Q_{3} / Q_{2}^{2}\right)(1 / \bar{N} a o)} \\
& {\left[C\left(\overline{\delta^{2}}\right)^{*}\right]^{2}=(6 / 5)\left(Q_{5} / Q_{3}{ }^{2}\right)(1 / \bar{N} a o) .}
\end{aligned}
$$

Intraregional errors of $N \lambda o, \bar{\lambda}$ and $\left(\overline{\lambda^{2}}\right)$ can be derived in the same way, starting from (3) and using (8). In practical measurements, a number of test lines have to be drawn on a test plane in order to yield a sufficiently large number of chords. Parallel test lines with regular and narrow intervals would be effective in minimizing intraregional errors. The mathematical treatments are, however, not simple, so that they are to be discussed as an independent problem in a forthcoming report. In this connection $M$ test lines are supposed to be randomly applied to the test plane. The errors as the square of coefficient of variation are then reduced to one $M$-th of those with a single test line of unit length. Provided that the region of measurement is sufficiently large, intraregional errors of $N \lambda o, \bar{\lambda}$ and $\left(\overline{\lambda^{2}}\right)$ are given by

and

$$
\begin{aligned}
& {[C(N \lambda o)]_{w}{ }^{2}=1 / M \bar{N} \lambda o} \\
& {[C(\bar{\lambda})]_{w}{ }^{2}=(9 / 8)\left(Q_{4} Q_{2} / Q_{3}{ }^{2}\right)(1 / M \bar{N} \lambda o)} \\
& {\left[C\left(\overline{\lambda^{2}}\right)\right]_{w}{ }^{2}=(4 / 3)\left(Q_{6} Q_{2} / Q_{4}{ }^{2}\right)(1 / M \bar{N} \lambda o) .}
\end{aligned}
$$

When $M$ is small, these expressions may be used in these forms immediately for the errors in reference to the total region. In the case of large $M$, intraregional errors are so reduced that interregional errors of $(20),(21)$ and (22) must be added to (39), (40) and (41), respectively, to express the errors for the total region.

Errors of Estimated Parameters. In the previous report of Suwa et al. (1976), the geometrical parameter of a distribution function was estimated from the ratio $\left(\overline{\delta^{2}}\right) / \bar{\delta}^{2}$ or $\left(\overline{\lambda^{2}}\right) / \bar{\lambda}^{2}$. The ratio is now simply expressed by $W$, while $\delta$ and $\lambda$ are likewise expressed with a common symbol $X$. There is a perfect positive correlation between $\left(\overline{X^{2}}\right)$ and $\bar{X}^{2}$, as indicated by (13), (14), (15) and (16), so that (A. 19) may be applied to the error of $W$, assuming $\rho$ is equal to $I$. Taking also (A. 15) into consideration, one can derive the error of $W$ as

$$
\left[C(W)^{*}\right]^{2}=\left[C\left(\overline{X^{2}}\right)^{*}-2 C(\bar{X})^{*}\right]^{2} \text {. }
$$

The error of scalar parameter $r_{0}$ and that of the total number $N v o$ of spheres in a unit volume can be stepwise derived, when the error of geometrical parameter is obtained. They must be calculated separately in individual distribution 
functions. However, the principle is essentially the same, and (A. 18) and (A. 19) are applied with pertinent evaluation of the coefficient of correlation. The process of calculation is explained to some extent in the subsequent part of this report.

\section{Application of the Derivatrons of Errors}

The mathematical derivations for errors are now applied to the parameters estimated from measurements on pancreatic islets in the previous report of Suwa et al. (1976).

\section{1) With $\delta$}

The estimated parameters are summarized as follows.

Gamma distribution Weibull distribution

Logarithmic

$$
\begin{array}{lll}
m=2.6206 & m=1.4905 & m=0.49385 \\
r_{0}=0.00877 \mathrm{~mm} & r_{0}=0.02397 \mathrm{~mm} & r_{0}=0.02203 \mathrm{~mm} \\
\bar{r}=0.02299 \mathrm{~mm} & \bar{r}=0.02166 \mathrm{~mm} & \bar{r}=0.02489 \mathrm{~mm} \\
N v o=84.42 / \mathrm{mm}^{3} & N v o=89.60 / \mathrm{mm}^{3} & N v o=77.98 \mathrm{~mm}^{3}
\end{array}
$$

The total number of islet sections measured was 623 . The error of Nao is common to all the distribution functions on account of $(36)$ and given as

or

$$
\begin{aligned}
& {\left[C(N a O)^{*}\right]^{2}=1 / 623=0.0016051} \\
& C(N a O)^{*}=0.04006
\end{aligned}
$$

For the determination of errors of $\bar{\delta}$ and $\left(\overline{\delta^{2}}\right)$, the coefficients $Q_{3} / Q_{2}{ }^{2}$ and $Q_{5} / Q_{3}{ }^{2}$ are calculated with Table 1 in the report of Suwa et al. (1976) and with the values of estimated parameters. The errors of these two quantities together with those of $W$ or $\left(\overrightarrow{\delta^{2}}\right) / \bar{\delta}^{2}$ are summarized as follows.

Gamma distribution

$$
\begin{array}{ll}
{\left[C(\bar{\delta})^{*}\right]^{2}=0.0022139} & C(\bar{\delta})^{*}=0.04705 \\
{\left[C\left(\overline{\delta^{2}}\right)^{*}\right]^{2}=0.0042844} & C\left(\overline{\delta^{2}}\right)^{*}=0.06546 \\
{\left[C(W)^{*}\right]^{2}=0.0008202} & C(W)^{*}=0.02864
\end{array}
$$

Weibull distribution

$$
\begin{array}{ll}
{\left[C(\bar{\delta})^{*}\right]^{2}=0.0022140} & C(\bar{\delta})^{*}=0.04705 \\
{\left[C\left(\overline{\delta^{2}}\right)^{*}\right]^{2}=0.0040492} & C\left(\overline{\delta^{2}}\right)^{*}=0.06363 \\
{\left[C(W)^{*}\right]^{2}=0.0009248} & C(W)^{*}=0.03047
\end{array}
$$

Logarithmic normal distribution

$$
\begin{array}{ll}
{\left[C(\bar{\delta})^{*}\right]^{2}=0.0022139} & C(\bar{\delta})^{*}=0.04705 \\
{\left[C\left(\overline{\delta^{2}}\right)^{*}\right]^{2}=0.0051093} & C\left(\overline{\delta^{2}}\right)^{*}=0.07148 \\
{\left[C(W)^{*}\right]^{2}=0.0005117} & C(W)^{*}=0.02262
\end{array}
$$


From the error of $W$ it is now possible to determine the error of the geometrical parameter $m$ of each distribution function. In gamma and Weibull distributions, however, $m$ is subject to a very large variation with only a slight error of $W$, when $m$ is not much larger than 1 . It is therefore not pertinent to use (A. 12), because the result is not reliable enough, when $C(F)$ is large. Besides, $W$ is a function composed of gamma functions of $m$ in the Weibull distribution. An analytical solution of $m$ is impossible. In these two distribution functions, $m$ is therefore calculated by the use of the maximum and minimum $W_{\mathrm{S}}$ at $95 \%$ level or with $W[1 \pm 1.96 C(W)]$. The maximum and minimum values of $m, m(\max )$ and $m(\min )$, are numerically obtained with the minimum and maximum $W_{S}$, respectively, and are regarded as presenting the upper and lower limits of $m$ at $95 \%$ level. The ratio of $[m(\max )-$ $m(\min )] / 1.96[m(\max )+m(\min )]$ is then used for an approximation of $C(m)$.

After $C(m)$ is determined, the errors of the other parameters $r_{0}, \bar{r}$ and $N v o$ in gamma distribution are then calculated by stepwise application of (A. 18) and (A. 19). In the Weibull distribution the errors of $r_{0}$ and $\vec{r}$ must also be determined numerically with the aid of the table of gamma function. Individual steps of calculation may be deleted for gamma and Weibull distributions and only the results will be presented. The principle of calculation is better explained in logarithmic normal distribution, because analytical treatments are possible throughout the course of calculation. The errors of the estimated parameters are as follows.

$$
\begin{array}{cll}
\text { Gamma distribution } & \text { Weibull distribution } & \begin{array}{c}
\text { Logarithmic } \\
\text { normal distribution }
\end{array} \\
C(m)^{*}=0.17825 & C(m)^{*}=0.09608 & C(m)^{*}=0.04637 \\
C\left(r_{0}\right)^{*}=0.14045 & C\left(r_{0}\right)^{*}=0.08158 & C\left(r_{0}\right)^{*}=0.05801 \\
C(\bar{r})^{*}=0.03781 & C(\bar{r})^{*}=0.07093 & C(\bar{r})^{*}=0.04670 \\
C(N v o)^{*}=0.05509 & C(N v o)^{*}=0.08077 & C(N v o)^{*}=0.06153
\end{array}
$$

The steps of calculation of these errors are explained with logarithmic normal distribution. In this distribution

$$
W=\left(32 / 3 \pi^{2}\right) \cdot \exp \left(m^{2}\right)
$$

as demonstrated in our previous report. The solution of (45) is

$$
m=\left[\log \left(3 \pi^{2} / 32\right)+\log W\right]^{1 / 2} .
$$

Consequently,

$$
\mathrm{d} m / \mathrm{d} W=1 / 2 m W,
$$

and applying $(A, 12)$ one obtains

and

$$
\operatorname{Var}(m)=(1 / 2 m W)^{2} \operatorname{Var}(W)
$$

Because $C(W)^{*}$ is 0.02262 as shown at the bottom of page $177, C(m)^{*}$ is given as

$$
C(m)^{*}=0.04637 \text {. }
$$


From Table 1 of our previous report, $r_{0}$ is determined by

$$
r_{0}=(2 / \pi) \bar{\delta} \cdot \exp \left(-3 m^{2} / 2\right) .
$$

As $m$ and $\bar{\delta}$ may be regarded as independent of each other, (A. 12) is applied to (51) and

$$
\left[C\left(r_{0}\right)^{*}\right]^{2}=\left[C(\bar{\delta})^{*}\right]^{2}+9 m^{4}\left[C(m)^{*}\right]^{2}
$$

is calculated with the estimated values of the parameters. The result is

$$
C\left(r_{0}\right)^{*}=0.05801 \text {. }
$$

For the derivation of the error of $\bar{r}$, the following relation is used.

$$
\bar{r}=r_{0} \cdot \exp \left(m^{2} / 2\right)
$$

Substituting $z$ for $\exp \left(m^{2} / 2\right)$ and taking (A. 12) into consideration

$$
C(z)=m^{2} C(m)
$$

is obtained. Now, expression (51) indicates that there is a negative correlation between $m$ and $r_{0}$ and hence between $z$ and $r_{0}$, so that (A. 18) may be applied to (54) with $\rho=-1$, provided that the variation of $m$ is a limited one. On account of (54) and (55) and because $m$ is 0.49385

$$
\begin{aligned}
C(\bar{r})^{*} & =\left|C\left(r_{0}\right)^{*}-C(z)^{*}\right|=\left|C\left(r_{0}\right)^{*}-\mathrm{m}^{2} C(m)^{*}\right| \\
& =0.04670
\end{aligned}
$$

is immediately obtained.

Finally, the error of $N v o$ is calculated from

$$
\left[C(N v o)^{*}\right]^{2}=\left[C(N \alpha o)^{*}\right]^{2}+\left[C(\bar{r})^{*}\right]^{2}
$$

as

$$
C(N v o)^{*}=0.06153
$$

2) With $\lambda$

The derivation of the errors for the parameters estimated by means of chord length is essentially the same as in the case of $\delta$. Further explanations on the derivation seem unnecessary and only the results of calculation will be presented. Firstly, the values of estimated parameters are reproduced from our previous report.

$$
\begin{array}{lll}
\text { Gamma distribution } & \text { Weibull distribution } & \begin{array}{c}
\text { Logarithmic } \\
\text { normal distribution }
\end{array} \\
m=0.93389 & m=0.98337 & m=0.54157 \\
r_{0}=0.0133 \mathrm{~mm} & r_{0}=0.0126 \mathrm{~mm} & r_{0}=0.0188 \mathrm{~mm} \\
\bar{r}=0.0125 \mathrm{~mm} & \bar{r}=0.0129 \mathrm{~mm} & \bar{r}=0.0218 \mathrm{~mm} \\
N v o=222.93 / \mathrm{mm}^{3} & N v o=213.09 / \mathrm{mm}^{3} & N v o=113.20 / \mathrm{mm}^{3}
\end{array}
$$


The total number of measured chords was 361 , and the total length of test lines was $1593.035 \mathrm{~mm}$. The region $H_{A}$ of measurement was $477.91 \mathrm{~mm}^{2}$ in surface area and equivalent to a square with sides of $\sqrt{H_{A}}$ or $21.8612 \mathrm{~mm}$ in length. The expectation $\bar{M}$ of the number of test lines covering the square is then

$$
\bar{M}=1593.035 / 21.8612=72.87 \text {. }
$$

The mean number of chords was $0.22661 / \mathrm{mm}$ and $\bar{\lambda}$ was $5.216 \times 10^{-2} \mathrm{~mm}$. These quantities are now expressed taking $21.8612 \mathrm{~mm}$ as the unit length as

$$
\bar{N} \lambda_{0}=4.95396
$$

and

$$
\bar{\lambda}=2.38597 \times 10^{-3}
$$

in the calculation of errors. Becasue $\bar{M}$ is large, the sums of intraregional and interregional errors are used for the errors of the total region.

The error of $N \lambda_{0}$ is independent of distribution functions and is given by

$$
\begin{aligned}
{\left[C(N \lambda o)^{*}\right]^{2} } & =(1 / M \bar{N} \lambda o)+(4 / \pi)(\bar{\lambda} / \bar{N} \lambda o) \\
& =0.0027701+0.0006132=0.0033833 .
\end{aligned}
$$

The errors of $\bar{\lambda},\left(\overline{\lambda^{2}}\right)$ and $W$ are different according to assumed distribution functions and are as follows.

Gamma distribution

$$
\begin{aligned}
& {\left[C(\bar{\lambda})^{*}\right]^{2}=0.0041784+0.0003046=0.0044830} \\
& C(\bar{\lambda})^{*}=0.06696 \\
& {\left[C\left(\overline{\lambda^{2}}\right)^{*}\right]^{2}=0.0093692+0.0012183=0.0105875} \\
& C\left(\overline{\lambda^{2}}\right)^{*}=0.10290 \\
& C(W)^{*}=0.03102
\end{aligned}
$$

Weibull distribution

$$
\begin{aligned}
{\left[C(\bar{\lambda})^{*}\right]^{2} } & =0.0041787+0.0002179=0.0043966 \\
C(\bar{\lambda})^{*} & =0.06631 \\
{\left[C\left(\overline{\lambda^{2}}\right)^{*}\right]^{2}=} & 0.0093965+0.0008716=0.0102681 \\
C\left(\overline{\lambda^{2}}\right)^{*} & =0.10133 \\
C(W)^{*} & =0.03129
\end{aligned}
$$

Logarithmic normal distribution

$$
\begin{aligned}
& {\left[C(\bar{\lambda})^{*}\right]^{2}=0.0041787+0.0001901=0.0043688} \\
& C(\bar{\lambda})^{*}=0.06610 \\
& {\left[C\left(\overline{\lambda^{2}}\right)^{*}\right]^{2}=0.0119384+0.0007602=0.0126986} \\
& C\left(\overline{\lambda^{2}}\right)^{*}=0.11269 \\
& C(W)^{*}=0.01950
\end{aligned}
$$

The errors of the parameters for each distribution function are given below. 


$\begin{array}{clc}\text { Gamma distribution } & \text { Weibull distribution } & \begin{array}{c}\text { Logarithmic } \\ \text { normal distribution }\end{array} \\ C(m)^{*}=0.3415 & C(m)^{*}=0.0930 & C(m)^{*}=0.0332 \\ C\left(r_{0}\right)^{*}=0.1919 & C\left(r_{0}\right)^{*}=0.1585 & C\left(r_{0}\right)^{*}=0.0821 \\ C(\bar{r})^{*}=0.1496 & C(\bar{r})^{*}=0.1184 & C(\bar{r})^{*}=0.0724 \\ C(N v o)^{*}=0.3048 & C(N v o)^{*}=0.2439 & C(N v o)^{*}=0.1560\end{array}$

\section{Comments}

The errors of parameters estimated by means of $\delta$ are relatively small and may be compatible with practical requirements. The final step of calculation is the errors of $N v o$, and they are limited within a range of about 5 to $8 \%$, when they are expressed as the coefficients of variation. On the contrary, the errors of estimated parameters are generally too large in the results with $\lambda$. They exceed practically tolerable ranges. The difference is first of all due to the small number of measured chords. In the case of $\delta$, the number of circles measured was 623 , while that of chords was only 361. The expressions (36), (37), (38), (39), (40) and (41) demonstrate that intraregional errors are inversely proportional to the number of circles or chords measured. In order to avoid too large errors, attempts must be made first of all at increasing the number of measurements.

However, it may be practically difficult to obtain a sufficiently large number of chords, when the density of spherical bodies is relatively low, as it is the case of pancreatic islets. A device effective in reducing intraregional errors is the use of

TABLE 1. Important symbols

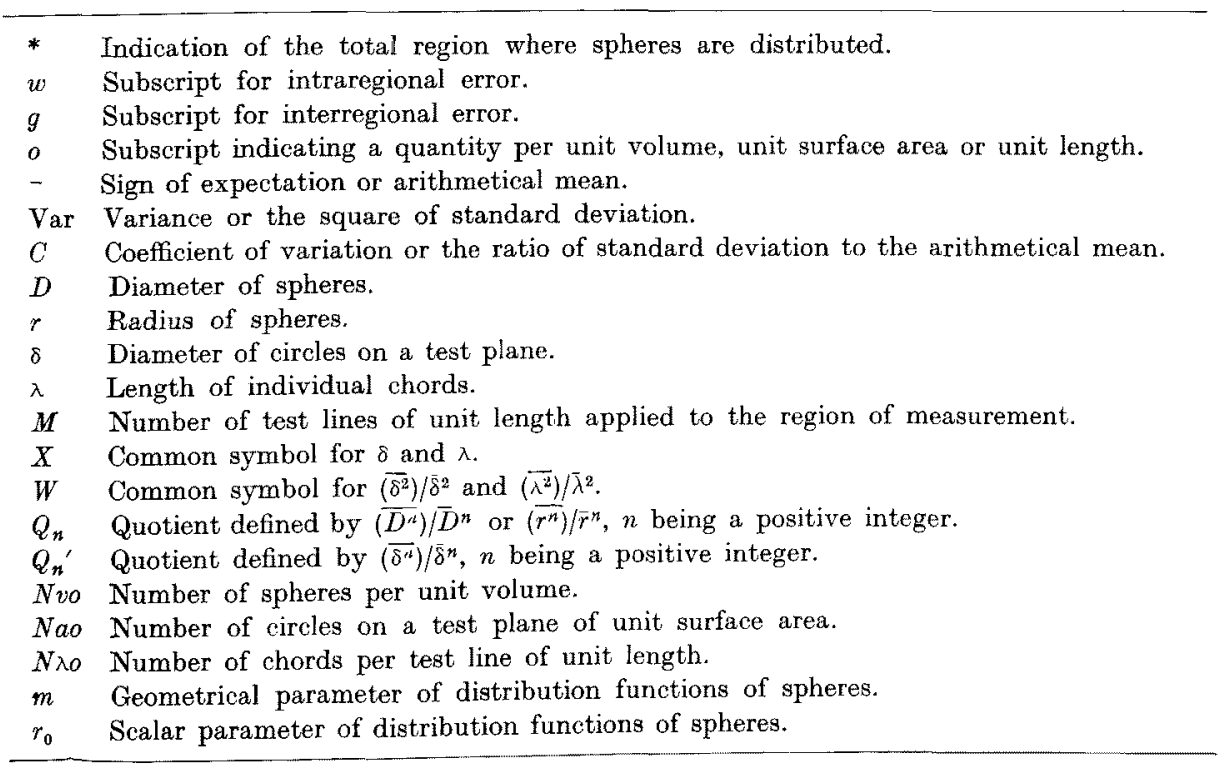


parallel test lines with uniform and narrow intervals. It will be revealed in a forthcoming report that the errors of $N \lambda o, \bar{\lambda}$ and $\overline{\left(\lambda^{2}\right)}$ are much smaller with such parallel test lines than with the same number of random ones.

Another factor which contributes to augmenting the errors of $N v o$ estimated from chords is the following relation,

$$
N v o=\bar{N} \lambda o / \pi r^{2} Q_{2} .
$$

The error of $N$ ro is consequently given by

$$
\left[C(N v o)^{*}\right]^{2}=\left[C(N \lambda o)^{*}\right]^{2}+4\left[C(\bar{r})^{*}\right]^{2} .
$$

It is easy to understand from (60) that the error of $\bar{\gamma}$ exerts an amplified effect upon the error of Nvo. This disadvantage is inherent to the method with chords and it is overcome only by sufficiently reducing intraregional errors of $\bar{\lambda}$ and $\overline{\left(\lambda^{2}\right)}$.

\section{APPENDIX}

The theorems on variance and coefficient of variation used in the present study are summarized.

1) Let $x$ be a random variable. The variance $\operatorname{Var}(x)$ of $x$ is given by

$$
\operatorname{Var}(x)=\left(\overline{x^{2}}\right)-\bar{x}^{2},
$$

$\left(\overline{x^{2}}\right)$ and $\bar{x}$ being the arithmetical means of $x^{2}$ and $x$, respectively; and the square of coefficient of variation $C(x)$ by

$$
C(x)^{2}=Q_{2}-1
$$

More general expressions are

and

$$
\begin{aligned}
& \operatorname{Var}\left(x^{n}\right)=\left(\overline{x^{2 n}}\right)-\left(\overline{x^{n}}\right)^{2} \\
& \mathrm{C}\left(x^{n}\right)^{2}=\left(Q_{2 n} / Q_{n}{ }^{2}\right)-1
\end{aligned}
$$

$n$ being a positive integer.

2) Let $k$ be a constant. The variance of $k x$ is then

$$
\operatorname{Var}(k x)=k^{2} \operatorname{Var}(x)
$$

and the coefficient of variation of $k x$ is

$$
\mathrm{C}(k x)=\mathrm{C}(x),
$$

or the coefficient of variation is not influenced by $k$.

3) Let $S$ be the sum of $m$ independent random variables or

$$
S=x_{1}+x_{2}+\cdots+x_{i}+\cdots+x_{m} .
$$

Then the variance of $S$ is given by

$$
\operatorname{Var}(S)=\sum_{i=1}^{m} \operatorname{Var}\left(x_{i}\right)
$$


or it is the sum of the variances of individual random variables, so far as they are independent of one another.

As a special case $x_{1}, x_{2}, \cdots, x_{i}, \cdots, x_{m}$ may be $m$ random samples from a common population of $x$. The mean $\bar{x}$ of $x$ is then equal to $S / m$. On account of (A. 8) and (A. 5) the following relations are derived.

$$
\begin{array}{ll} 
& \operatorname{Var}(S)=m \operatorname{Var}(x) \\
& \operatorname{Var}(\bar{x})=\operatorname{Var}(S) / m^{2}=\operatorname{Var}(x) / m \\
\text { and } \quad & \mathrm{C}(\bar{x})^{2}=\mathrm{C}(S)^{2}=\mathrm{C}(x)^{2} / m .
\end{array}
$$

4) Let $F$ or $F\left(x_{1}, x_{2}, \cdots, x_{i}, \cdots, x_{m}\right)$ be a function of $m$ independent random variables. The variance of $F$ is approximately

$$
\operatorname{Var}(F)=\sum_{i=1}^{m}\left(\partial F / \partial x_{i}\right)^{2} \operatorname{Var}\left(x_{i}\right) .
$$

If $F$ is the function of a random variable $x$ in the form of

$$
F=k x^{n}
$$

$k$ and $n$ being constants, $\operatorname{Var}(F)$ is given on account of (A. 12) as

$$
\operatorname{Var}(F)=\left(k n x^{n-1}\right)^{2} \operatorname{Var}(x),
$$

and $\mathrm{C}(F)^{2}$ as

$$
\mathrm{C}(F)^{2}=n^{2} \mathrm{C}(x)^{2}
$$

5) Let $F$ and $G$ be respectively the product and ratio of two random variables $x$ and $y$ or

$$
\begin{aligned}
& F=x y \\
& G=x / y .
\end{aligned}
$$

The coefficients of variation for $F$ and $G$ are determined approximately by

and

$$
\begin{aligned}
& \mathrm{C}(F)^{2}=\mathrm{C}(x)^{2}+\mathrm{C}(y)^{2}+2 \rho \mathrm{C}(x) \mathrm{C}(y) \\
& \mathrm{C}(G)^{2}=\mathrm{C}(x)^{2}+\mathrm{C}(y)^{2}-2 \rho \mathrm{C}(x) \mathrm{C}(y),
\end{aligned}
$$

where $\rho$ is the correlation coefficient of $x$ and $y$. When $x$ and $y$ are independent of each other, $\rho$ is 0 and one obtains

$$
\mathrm{C}(F)^{2}=\mathrm{C}(G)^{2}=\mathrm{C}(x)^{2}+\mathrm{C}(y)^{2} .
$$

In the case of perfect correlation of $x$ and $y, \rho$ is equal to \pm 1 , so that (A. 18) and (A. 19) become

and

$$
\begin{aligned}
& C(F)^{2}=[\mathrm{C}(x) \pm \mathrm{C}(y)]^{2} \\
& \mathrm{C}(G)^{2}=[\mathrm{C}(x) \mp \mathrm{C}(y)]^{2} .
\end{aligned}
$$

\section{Reference}

1) Suwa, N., Takahashi, T., Saito, K. \& Sawai, T. (1976) Morphometrical method to estimate the parameters of distribution functions assumed for spherical bodies from measurements on a random section. Tohoku J. exp. Med., 118, 101-111. 